PROCEEDINGS OF THE

AMERICAN MATHEMATICAL SOCIETY

Volume 135, Number 4, April 2007, Pages 951-959

S 0002-9939(06)08588-1

Article electronically published on September 26, 2006

\title{
FINITELY PRESENTABLE, NON-HOPFIAN GROUPS WITH KAZHDAN'S PROPERTY (T) AND INFINITE OUTER AUTOMORPHISM GROUP
}

\author{
YVES DE CORNULIER
}

(Communicated by Dan M. Barbasch)

\begin{abstract}
We give simple examples of Kazhdan groups with infinite outer automorphism groups. This answers a question of Paulin, independently answered by Ollivier and Wise by completely different methods. As arithmetic lattices in (non-semisimple) Lie groups, our examples are in addition finitely presented.

We also use results of Abels about compact presentability of $p$-adic groups to exhibit a finitely presented non-Hopfian Kazhdan group. This answers a question of Ollivier and Wise.
\end{abstract}

\section{INTRODUCTION}

Recall that a locally compact group is said to have Property (T) if every weakly continuous unitary representation with almost invariant vectors 11 has non-zero invariant vectors.

It was asked by Paulin in [HV, p. 134] (1989) whether there exists a group with Kazhdan's Property (T) and with an infinite outer automorphism group. This question remained unanswered until 2004; in particular, it is Question 18 in [Wo.

This question was motivated by the two following special cases. The first is the case of lattices in semisimple groups over local fields, which have long been considered as prototypical examples of groups with Property ( $T$ ). If $\Gamma$ is such a lattice, Mostow's rigidity theorem and the fact that semisimple groups have finite outer automorphism group imply that $\operatorname{Out}(\Gamma)$ is finite. Second, a new source of groups with Property $(\mathrm{T})$ appeared when Zuk $[\mathrm{Zu}$. proved that certain models of random groups have Property (T). But they are also hyperbolic, and Paulin proved Pa that a hyperbolic group with Property $(\mathrm{T})$ has a finite outer automorphism group.

However, it turns out that various arithmetic lattices in appropriate nonsemisimple groups provide examples. For instance, consider the additive group $\operatorname{Mat}_{m n}(\mathbf{Z})$ of $m \times n$ matrices over $\mathbf{Z}$, endowed with the action of $\mathrm{GL}_{n}(\mathbf{Z})$ by left multiplication.

Received by the editors February 25, 2005 and, in revised form, October 28, 2005.

2000 Mathematics Subject Classification. Primary 20F28; Secondary 20G25, 17 B56.

${ }^{1}$ A representation $\pi: G \rightarrow \mathcal{U}(\mathcal{H})$ almost has invariant vectors if for every $\varepsilon>0$ and every finite subset $F \subseteq G$, there exists a unit vector $\xi \in \mathcal{H}$ such that $\|\pi(g) \xi-\xi\|<\varepsilon$ for every $g \in F$. 
Proposition 1.1. For every $n \geq 3, m \geq 1, \mathrm{SL}_{n}(\mathbf{Z}) \ltimes \operatorname{Mat}_{m n}(\mathbf{Z})$ is a finitely presented linear group, has Property $(\mathrm{T})$, is non-coHopfian 2 and its outer automorphism group contains a copy of $\mathrm{PGL}_{m}(\mathbf{Z})$, hence is infinite if $m \geq 2$.

We later learned that Ollivier and Wise $\mathrm{OW}$ had independently found examples of a very different nature. They embed any countable group $G$ in $\operatorname{Out}(\Gamma)$, where $\Gamma$ has Property $(\mathrm{T})$, and is a subgroup of a torsion-free hyperbolic group, satisfying a certain "graphical" small cancellation condition (see also BS] ). In contrast to our examples, theirs are not, a priori, finitely presented; on the other hand, our examples are certainly not subgroups of hyperbolic groups since they all contain a copy of $\mathbf{Z}^{2}$.

They also construct in $\mathrm{OW}$ a non-coHopfian group with Property $(\mathrm{T})$ that embeds in a hyperbolic group. Proposition 1.1 actually answers two questions in their paper: namely, whether there exists a finitely presented group with Property $(\mathrm{T})$ and without the coHopfian Property (resp. with infinite outer automorphism group).

Remark 1.2. Another example of non-coHopfian group with Property (T) is $\mathrm{PGL}_{n}\left(\mathbf{F}_{p}[X]\right)$ when $n \geq 3$. This group is finitely presentable if $n \geq 4$ [RS (but not for $n=3[\mathrm{Be}$ ). In contrast with the previous examples, the Frobenius morphism Fr induces an isomorphism onto a subgroup of infinite index.

Ollivier and Wise also constructed in $\mathrm{OW}$ the first examples of non-Hopfian groups with Property (T). They asked whether a finitely presented example exists. Although linear finitely generated groups are residually finite, hence Hopfian, we use them to answer positively their question.

Theorem 1.3. There exists a $S$-arithmetic lattice $\Gamma$, and a central subgroup $Z \subset \Gamma$, such that $\Gamma$ and $\Gamma / Z$ are finitely presented, have Property $(\mathrm{T})$, and $\Gamma / Z$ is nonHopfian.

The group $\Gamma$ has a simple description as a matrix group from which Property $(\mathrm{T})$ and the non-Hopfian property for $\Gamma / Z$ are easily checked (Proposition 2.7). Section 3 is devoted to prove finite presentability of $\Gamma$. Here we use a general criterion for finite presentability of $S$-arithmetic groups, due to Abels A2. It involves the computation of the first and second cohomology group of a suitable Lie algebra.

\section{Proofs of all Results eXCept finite PRESEntability of $\Gamma$}

We need some facts about Property $(\mathrm{T})$.

Lemma 2.1 (see [HV] Chap. 3, Théorème 4]). Let $G$ be a locally compact group, and $\Gamma$ a lattice in $G$. Then $G$ has Property $(\mathrm{T})$ if and only if $\Gamma$ has Property $(\mathrm{T})$.

The next lemma is an immediate consequence of the classification of semisimple algebraic groups over local fields with Property (T) (see Ma, Chap. III, Theorem 5.6]) and S. P. Wang's results on the non-semisimple case [Wa, Theorem 2.10].

Lemma 2.2. Let $\mathbf{K}$ be a local field and $G$ a connected linear algebraic group defined over $\mathbf{K}$. Suppose that $G$ is perfect, and, for every simple quotient $S$ of $G$, either $S$ has $\mathbf{K}$-rank $\geq 2$, or $\mathbf{K}=\mathbf{R}$ and $S$ is isogeneous to either $\operatorname{Sp}(n, 1)(n \geq 2)$

\footnotetext{
${ }^{2}$ A group is coHopfian (resp. Hopfian) if it is isomorphic to no proper subgroup (resp. quotient)
} of itself. 
or $\mathrm{F}_{4(-20)}$. If $\operatorname{char}(\mathbf{K})>0$, suppose in addition that $G$ has a Levi decomposition defined over $\mathbf{K}$. Then $G(\mathbf{K})$ has Property $(\mathrm{T})$.

Proof of Proposition 1.1. The group $\mathrm{SL}_{n}(\mathbf{Z}) \ltimes \operatorname{Mat}_{m n}(\mathbf{Z})$ is linear in dimension $n+$ $m$. As a semidirect product of two finitely presented groups, it is finitely presented. For every $k \geq 2$, it is isomorphic to its proper subgroup $\mathrm{SL}_{n}(\mathbf{Z}) \ltimes k \mathrm{Mat}_{m n}(\mathbf{Z})$ of finite index $k^{m n}$.

The group $\mathrm{GL}_{m}(\mathbf{Z})$ acts on $\operatorname{Mat}_{m n}(\mathbf{Z})$ by right multiplication. Since this action commutes with the left multiplication of $\mathrm{SL}_{n}(\mathbf{Z})$, the group $\mathrm{GL}_{m}(\mathbf{Z})$ acts on the semidirect product $\mathrm{SL}_{n}(\mathbf{Z}) \ltimes \operatorname{Mat}_{m n}(\mathbf{Z})$ by automorphisms, and, by an immediate verification, this gives an embedding of $\mathrm{GL}_{m}(\mathbf{Z})$ if $n$ is odd or $\mathrm{PGL}_{m}(\mathbf{Z})$ if $n$ is even into $\operatorname{Out}\left(\mathrm{SL}_{n}(\mathbf{Z}) \ltimes \operatorname{Mat}_{m n}(\mathbf{Z})\right.$ ) (it can be shown that this is an isomorphism if $n$ is odd; if $n$ is even, the image has index two). In particular, if $m \geq 2$, then $\mathrm{SL}_{n}(\mathbf{Z}) \ltimes \operatorname{Mat}_{m n}(\mathbf{Z})$ has an infinite outer automorphism group.

On the other hand, in view of Lemma 2.1, it has Property (T) (actually for all $m \geq 0)$ : indeed, $\mathrm{SL}_{n}(\mathbf{Z}) \ltimes \operatorname{Mat}_{m n}(\mathbf{Z})$ is a lattice in $\mathrm{SL}_{n}(\mathbf{R}) \ltimes \operatorname{Mat}_{m n}(\mathbf{R})$, which has Property (T) by Lemma 2.2 as $n \geq 3$.

We now turn to the proof of Theorem 1.3 . The following lemma is immediate, and already used in $\mathrm{Ha}$ and $\mathrm{A1}$.

Lemma 2.3. Let $\Gamma$ be a group and $Z$ a central subgroup. Let $\alpha$ be an automorphism of $\Gamma$ such that $\alpha(Z)$ is a proper subgroup of $Z$. Then $\alpha$ induces a surjective, noninjective endomorphism of $\Gamma / Z$, whose kernel is $\alpha^{-1}(Z) / Z$.

Definition 2.4. Fix $n_{1}, n_{2}, n_{3}, n_{4} \in \mathbf{N}-\{0\}$ with $n_{2}, n_{3} \geq 3$. We set $\Gamma=$ $G(\mathbf{Z}[1 / p])$, where $p$ is any prime, and $G$ is the algebraic group defined as matrices by blocks of size $n_{1}, n_{2}, n_{3}, n_{4}$ :

$$
\left(\begin{array}{cccc}
I_{n_{1}} & (*)_{12} & (*)_{13} & (*)_{14} \\
0 & (* *)_{22} & (*)_{23} & (*)_{24} \\
0 & 0 & (*)_{33} & (*)_{34} \\
0 & 0 & 0 & I_{n_{4}}
\end{array}\right)
$$

where $(*)$ denote any matrices and $(* *)_{i i}$ denotes matrices in $\mathrm{SL}_{n_{i}}, i=2,3$.

The centre of $G$ consists of matrices of the form

$$
\left(\begin{array}{cccc}
I_{n_{1}} & 0 & 0 & (*)_{14} \\
0 & I_{n_{2}} & 0 & 0 \\
0 & 0 & I_{n_{3}} & 0 \\
0 & 0 & 0 & I_{n_{4}}
\end{array}\right) .
$$

Define $Z$ as the centre of $G(\mathbf{Z})$.

Remark 2.5. This group is related to an example due to Abels: in [A1 he considers the same group, but with blocks $1 \times 1$, and $\mathrm{GL}_{1}$ instead of $\mathrm{SL}_{1}$ in the diagonal. Taking the points over $\mathbf{Z}[1 / p]$, and taking the quotient by a cyclic subgroup of the centre, this provided the first example of a finitely presentable non-Hopfian solvable group.

Remark 2.6. If we do not care about finite presentability, we can take $n_{3}=0$ (i.e. 3 blocks suffice). 
We begin by easy observations. Identify $\mathrm{GL}_{n_{1}}$ to the upper left diagonal block. It acts by conjugation on $G$ as follows:

$$
\left(\begin{array}{cccc}
u & 0 & 0 & 0 \\
0 & I & 0 & 0 \\
0 & 0 & I & 0 \\
0 & 0 & 0 & I
\end{array}\right) \cdot\left(\begin{array}{cccc}
I & A_{12} & A_{13} & A_{14} \\
0 & B_{2} & A_{23} & A_{24} \\
0 & 0 & B_{3} & A_{34} \\
0 & 0 & 0 & I
\end{array}\right)=\left(\begin{array}{cccc}
I & u A_{12} & u A_{13} & u A_{14} \\
0 & B_{2} & A_{23} & A_{24} \\
0 & 0 & B_{3} & A_{34} \\
0 & 0 & 0 & I
\end{array}\right) .
$$

This gives an action of $\mathrm{GL}_{n_{1}}$ on $G$, and also on its centre, and this latter action is faithful. In particular, for every commutative ring $R, \mathrm{GL}_{n_{1}}(R)$ embeds in $\operatorname{Out}(G(R))$.

From now on, we suppose that $R=\mathbf{Z}[1 / p]$, and $u=p I_{n_{1}}$. The automorphism of $\Gamma=G(\mathbf{Z}[1 / p])$ induced by $u$ maps $Z$ to its proper subgroup $Z^{p}$. In view of Lemma 2.3. this implies that $\Gamma / Z$ is non-Hopfian.

Proposition 2.7. The groups $\Gamma$ and $\Gamma / Z$ are finitely generated, have Property $(\mathrm{T})$, and $\Gamma / Z$ is non-Hopfian.

Proof. We have just proved that $\Gamma / Z$ is non-Hopfian. By the Borel-Harish-Chandra Theorem [BHC], $\Gamma$ is a lattice in $G(\mathbf{R}) \times G\left(\mathbf{Q}_{p}\right)$. This group has Property (T) as a consequence of Lemma 2.2. By Lemma 2.1, $\Gamma$ also has Property $(\mathrm{T})$. Finite generation is a consequence of Property (T) [HV, Lemme 10]. Since Property (T) is (trivially) inherited by quotients, $\Gamma / Z$ also has Property $(\mathrm{T})$.

Remark 2.8. This group has a surjective endomorphism with a non-trivial finite kernel. We have no analogous example with an infinite kernel. Such examples might be constructed if we could prove that some groups over rings of dimension $\geq 2$ such as $\mathrm{SL}_{n}(\mathbf{Z}[X])$ or $\mathrm{SL}_{n}\left(\mathbf{F}_{p}[X, Y]\right)$ have Property (T), but this is an open problem $\underline{\mathrm{Sh}}$. The non-Hopfian Kazhdan group of Ollivier and Wise $\mathrm{OW}$ is torsion-free, so the kernel is infinite in their case.

Remark 2.9. It is easy to check that $\mathrm{GL}_{n_{1}}(\mathbf{Z}) \times \mathrm{GL}_{n_{4}}(\mathbf{Z})$ embeds in $\operatorname{Out}(\Gamma)$ and $\operatorname{Out}(\Gamma / Z)$. In particular, if $\max \left(n_{1}, n_{2}\right) \geq 2$, then these outer automorphism groups are infinite.

We finish this section by observing that $Z$ is a finitely generated subgroup of the centre of $\Gamma$, so that finite presentability of $\Gamma / Z$ immediately follows from that of $\Gamma$.

\section{Finite presentability of $\Gamma$}

We recall that a Hausdorff topological group $H$ is compactly presented if there exists a compact generating subset $C$ of $H$ such that the abstract group $H$ is the quotient of the group freely generated by $C$ by relations of bounded length. See [A2, §1.1] for more about compact presentability.

Kneser $[\mathrm{Kn}]$ has proved that for every linear algebraic $\mathbf{Q}_{p}$-group, the $S$-arithmetic lattice $G(\mathbf{Z}[1 / p])$ is finitely presented if and only if $G\left(\mathbf{Q}_{p}\right)$ is compactly presented. A characterization of the linear algebraic $\mathbf{Q}_{p}$-groups $G$ such that $G\left(\mathbf{Q}_{p}\right)$ is compactly presented is given in A2. This criterion requires the study of a solvable cocompact subgroup of $G\left(\mathbf{Q}_{p}\right)$, which seems hard to carry out in our specific example.

Let us describe another sufficient criterion for compact presentability, also given in A2, which is applicable to our example. Let $U$ be the unipotent radical in $G$, and let $S$ denote a Levi factor defined over $\mathbf{Q}_{p}$, so that $G=S \ltimes U$. Let $\mathfrak{u}$ be the 
Lie algebra of $U$, and let $D$ be a maximal $\mathbf{Q}_{p}$-split torus in $S$. We recall that the first homology group of $\mathfrak{u}$ is defined as the abelianization

$$
H_{1}(\mathfrak{u})=\mathfrak{u} /[\mathfrak{u}, \mathfrak{u}],
$$

and the second homology group of $\mathfrak{u}$ is defined as $\operatorname{Ker}\left(d_{2}\right) / \operatorname{Im}\left(d_{3}\right)$, where the maps

$$
\mathfrak{u} \wedge \mathfrak{u} \wedge \mathfrak{u} \stackrel{d_{3}}{\rightarrow} \mathfrak{u} \wedge \mathfrak{u} \stackrel{d_{2}}{\rightarrow} \mathfrak{u}
$$

are defined by

$d_{2}\left(x_{1} \wedge x_{2}\right)=-\left[x_{1}, x_{2}\right]$ and $d_{3}\left(x_{1} \wedge x_{2} \wedge x_{3}\right)=x_{3} \wedge\left[x_{1}, x_{2}\right]+x_{2} \wedge\left[x_{3}, x_{1}\right]+x_{1} \wedge\left[x_{2}, x_{3}\right]$.

We can now state the result by Abels that we use (see A2, Theorem 6.4.3 and Remark 6.4.5]).

Theorem 3.1. Let $G$ be a connected linear algebraic group over $\mathbf{Q}_{p}$. Suppose that the following assumptions are fulfilled:

(i) $G$ is $\mathbf{Q}_{p}$-split.

(ii) $G$ has no simple quotient of $\mathbf{Q}_{p}$-rank one.

(iii) 0 does not lie on the segment joining two dominant weights for the adjoint representation of $S$ on $H_{1}(\mathfrak{u})$.

(iv) 0 is not a dominant weight for an irreducible subrepresentation of the adjoint representation of $\mathrm{S}$ on $\mathrm{H}_{2}(\mathfrak{u})$.

Then $G\left(\mathbf{Q}_{p}\right)$ is compactly presented.

We now return to our particular example of $G$, observe that it is clearly $\mathbf{Q}_{p}$-split, and that its simple quotients are $\mathrm{SL}_{n_{2}}$ and $\mathrm{SL}_{n_{3}}$, which have rank greater than one. Keep the previous notations $S, D, U, \mathfrak{u}$, so that $S$ (resp. $D$ ) denotes in our case the diagonal-by-blocks (resp. diagonal) matrices in $G$, and $U$ denotes the matrices in $G$ all of whose diagonal blocks are the identity. The set of indices of the matrix is partitioned as $I=I_{1} \sqcup I_{2} \sqcup I_{3} \sqcup I_{4}$, with $\left|I_{j}\right|=n_{j}$ as in Definition 2.4. It follows that, for every field $K$,

$$
\mathfrak{u}(K)=\left\{T \in \operatorname{End}\left(K^{I}\right), \forall j, T\left(K^{I_{j}}\right) \subset \bigoplus_{i<j} K^{I_{i}}\right\} .
$$

Throughout, we use the following notation: a letter such as $i_{k}$ (or $j_{k}$, etc.) implicitly means $i_{k} \in I_{k}$. Define, in an obvious way, subgroups $U_{i j}, i<j$, of $U$, and their Lie algebras $\mathfrak{u}_{i j}$.

We begin by checking condition (iii) of Theorem 3.1

Lemma 3.2. For any two weights of the action of $D$ on $H_{1}(\mathfrak{u}), 0$ is not on the segment joining them.

Proof. Recall that $H_{1}(\mathfrak{u})=\mathfrak{u} /[\mathfrak{u}, \mathfrak{u}]$. So it suffices to look at the action on the supplement $D$-subspace $\mathfrak{u}_{12} \oplus \mathfrak{u}_{23} \oplus \mathfrak{u}_{34}$ of $[\mathfrak{u}, \mathfrak{u}]$. Identifying $S$ with $\mathrm{SL}_{n_{2}} \times \mathrm{SL}_{n_{3}}$, let us denote by $(A, B)$ an element of $D \subset S$. We also denote by $e_{p q}$ the matrix whose coefficient $(p, q)$ equals one, and all others are zero.

$$
(A, B) \cdot e_{i_{1} j_{2}}=a_{j_{2}}^{-1} e_{i_{1} j_{2}}, \quad(A, B) \cdot e_{j_{2} k_{3}}=a_{j_{2}} b_{k_{3}}^{-1} e_{j_{2} k_{3}}, \quad(A, B) \cdot e_{k_{3} \ell_{4}}=b_{k_{3}} e_{k_{3} \ell_{4}} .
$$

Since $S=\mathrm{SL}_{n_{2}} \times \mathrm{SL}_{n_{3}}$, the weights for the adjoint action on $\mathfrak{u}_{12} \oplus \mathfrak{u}_{23} \oplus \mathfrak{u}_{34}$ live in $M / P$, where $M$ is the free $\mathbf{Z}$-module of rank $n_{2}+n_{3}$ with basis $\left(u_{1}, \ldots, u_{n_{2}}\right.$, 
$\left.v_{1}, \ldots, v_{n_{3}}\right)$, and $P$ is the plane generated by $\sum_{j_{2}} u_{j_{2}}$ and $\sum_{k_{3}} v_{k_{3}}$. Thus, the weights are (modulo $P)-u_{j_{2}}, u_{j_{2}}-v_{k_{3}}, v_{k_{3}}\left(1 \leq j_{2} \leq n_{2}, 1 \leq k_{3} \leq n_{3}\right)$.

Using that $n_{2}, n_{3} \geq 3$, it is clear that no non-trivial positive combination of two weights (viewed as elements of $\mathbf{Z}^{n_{2}+n_{3}}$ ) lies in $P$.

We must now check condition (iv) of Theorem 3.1 and therefore compute $H_{2}(\mathfrak{u})$ as a $D$-module.

Lemma 3.3. $\operatorname{Ker}\left(d_{2}\right)$ is generated by

(1) $\mathfrak{u}_{12} \wedge \mathfrak{u}_{12}, \mathfrak{u}_{23} \wedge \mathfrak{u}_{23}, \mathfrak{u}_{34} \wedge \mathfrak{u}_{34}, \mathfrak{u}_{13} \wedge \mathfrak{u}_{23}, \mathfrak{u}_{23} \wedge \mathfrak{u}_{24}, \mathfrak{u}_{12} \wedge \mathfrak{u}_{13}, \mathfrak{u}_{24} \wedge \mathfrak{u}_{34}$, $\mathfrak{u}_{12} \wedge \mathfrak{u}_{34}$.

(2) $\mathfrak{u}_{14} \wedge \mathfrak{u}, \mathfrak{u}_{13} \wedge \mathfrak{u}_{13}, \mathfrak{u}_{24} \wedge \mathfrak{u}_{24}, \mathfrak{u}_{13} \wedge \mathfrak{u}_{24}$.

(3) $e_{i_{1} j_{2}} \wedge e_{k_{2} \ell_{3}}\left(j_{2} \neq k_{2}\right), e_{i_{2} j_{3}} \wedge e_{k_{3} \ell_{4}}\left(j_{3} \neq \ell_{3}\right)$.

(4) $e_{i_{1} j_{2}} \wedge e_{k_{2} \ell_{4}}\left(j_{2} \neq k_{2}\right), e_{i_{1} j_{3}} \wedge e_{k_{3} \ell_{4}}\left(j_{3} \neq k_{3}\right)$.

(5) Elements of the form $\sum_{j_{2}} \alpha_{j_{2}}\left(e_{i_{1} j_{2}} \wedge e_{j_{2} k_{3}}\right)$ if $\sum_{j_{2}} \alpha_{j_{2}}=0$, and $\sum_{j_{3}} \alpha_{j_{3}}\left(e_{i_{2} j_{3}} \wedge e_{j_{3} k_{4}}\right)$ if $\sum_{j_{3}} \alpha_{j_{3}}=0$.

(6) Elements of the form $\sum_{j_{2}} \alpha_{j_{2}}\left(e_{i_{1} j_{2}} \wedge e_{j_{2} k_{4}}\right)+\sum_{j_{3}} \beta_{j_{3}}\left(e_{i_{1} j_{3}} \wedge e_{j_{3} k_{4}}\right)$ if $\sum_{j_{2}} \alpha_{j_{2}}$ $+\sum_{j_{3}} \beta_{j_{3}}=0$.

Proof. First observe that $\operatorname{Ker}\left(d_{2}\right)$ contains $\mathfrak{u}_{i j} \wedge \mathfrak{u}_{k l}$ when $\left[\mathfrak{u}_{i j}, \mathfrak{u}_{k l}\right]=0$. This corresponds to (1) and (2). The remaining cases are $\mathfrak{u}_{12} \wedge \mathfrak{u}_{23}, \mathfrak{u}_{23} \wedge \mathfrak{u}_{34}, \mathfrak{u}_{12} \wedge \mathfrak{u}_{24}$, $\mathfrak{u}_{13} \wedge \mathfrak{u}_{34}$.

On the one hand, $\operatorname{Ker}\left(d_{2}\right)$ also contains $e_{i_{1} j_{2}} \wedge e_{k_{2} \ell_{3}}$ if $j_{2} \neq k_{2}$, etc.; this corresponds to elements in (3), (4). On the other hand, $d_{2}\left(e_{i_{1} j_{2}} \wedge e_{j_{2} k_{3}}\right)=-e_{i_{1} k_{3}}$, $d_{2}\left(e_{i_{2} j_{3}} \wedge e_{j_{3} k_{4}}\right)=-e_{i_{2} k_{4}}, d_{2}\left(e_{i_{1} j_{2}} \wedge e_{j_{2} k_{4}}\right)=-e_{i_{1} k_{4}}, d_{2}\left(e_{i_{1} j_{3}} \wedge e_{j_{3} k_{4}}\right)=-e_{i_{1} k_{4}}$. The lemma follows.

Definition 3.4. Denote by $\mathfrak{b}$ (resp. $\mathfrak{h}$ ) the subspace generated by elements in (2), (4), and (6) (resp. in (1), (3), and (5)) of Lemma 3.3.

Proposition 3.5. $\operatorname{Im}\left(d_{3}\right)=\mathfrak{b}$, and $\operatorname{Ker}\left(d_{2}\right)=\mathfrak{b} \oplus \mathfrak{h}$ as a D-module. In particular, $\mathrm{H}_{2}(\mathfrak{u})$ is isomorphic to $\mathfrak{h}$ as a D-module.

Proof. We first prove, in a series of facts, that $\operatorname{Im}\left(d_{3}\right) \supset \mathfrak{b}$.

Fact. $\mathfrak{u}_{14} \wedge \mathfrak{u}$ is contained in $\operatorname{Im}\left(d_{3}\right)$.

Proof. If $z \in \mathfrak{u}_{14}$, then $d_{3}(x \wedge y \wedge z)=z \wedge[x, y]$. This already shows that $\mathfrak{u}_{14} \wedge$ $\left(\mathfrak{u}_{13} \oplus \mathfrak{u}_{24} \oplus \mathfrak{u}_{14}\right)$ is contained in $\operatorname{Im}\left(d_{3}\right)$, since $[\mathfrak{u}, \mathfrak{u}]=\mathfrak{u}_{13} \oplus \mathfrak{u}_{24} \oplus \mathfrak{u}_{14}$.

Now, if $(x, y, z) \in \mathfrak{u}_{24} \times \mathfrak{u}_{12} \times \mathfrak{u}_{34}$, then $d_{3}(x \wedge y \wedge z)=z \wedge[x, y]$. Since $\left[\mathfrak{u}_{24}, \mathfrak{u}_{12}\right]=$ $\mathfrak{u}_{14}$, this implies that $\mathfrak{u}_{14} \wedge \mathfrak{u}_{34} \subset \operatorname{Im}\left(d_{3}\right)$. Similarly, $\mathfrak{u}_{14} \wedge \mathfrak{u}_{12} \subset \operatorname{Im}\left(d_{3}\right)$.

Finally we must prove that $\mathfrak{u}_{14} \wedge \mathfrak{u}_{23} \subset \operatorname{Im}\left(d_{3}\right)$. This follows from the formula $e_{i_{1} j_{4}} \wedge e_{k_{2} \ell_{3}}=d_{3}\left(e_{i_{1} m_{2}} \wedge e_{k_{2} \ell_{3}} \wedge e_{m_{2} j_{4}}\right)$, where $m_{2} \neq k_{2}$ (so that we use that $\left.\left|I_{2}\right| \geq 2\right)$.

Fact. $\mathfrak{u}_{13} \wedge \mathfrak{u}_{13}$ and, similarly, $\mathfrak{u}_{24} \wedge \mathfrak{u}_{24}$, are contained in $\operatorname{Im}\left(d_{3}\right)$.

Proof. If $(x, y, z) \in \mathfrak{u}_{12} \times \mathfrak{u}_{23} \times \mathfrak{u}_{13}$, then $d_{3}(x \wedge y \wedge z)=z \wedge[x, y]$. Since $\left[\mathfrak{u}_{12}, \mathfrak{u}_{23}\right]=$ $\mathfrak{u}_{13}$, this implies that $\mathfrak{u}_{13} \wedge \mathfrak{u}_{13} \subset \operatorname{Im}\left(d_{3}\right)$.

Fact. $\mathfrak{u}_{13} \wedge \mathfrak{u}_{24}$ is contained in $\operatorname{Im}\left(d_{3}\right)$.

Proof. $d_{3}\left(e_{i_{1} k_{2}} \wedge e_{k_{2} \ell_{3}} \wedge e_{k_{2} j_{4}}\right)=e_{k_{2} j_{4}} \wedge e_{i_{1} \ell_{3}}+e_{i_{1} j_{4}} \wedge e_{k_{2} \ell_{3}}$. Since we already know that $e_{i_{1} j_{4}} \wedge e_{k_{2} \ell_{3}} \in \operatorname{Im}\left(d_{3}\right)$, this implies $e_{k_{2} j_{4}} \wedge e_{i_{1} \ell_{3}} \in \operatorname{Im}\left(d_{3}\right)$. 
Fact. The elements in (4) are in $\operatorname{Im}\left(d_{3}\right)$.

Proof. $d_{3}\left(e_{i_{1} j_{2}} \wedge e_{j_{2} k_{3}} \wedge e_{\ell_{3} m_{4}}\right)=-e_{i_{1} k_{3}} \wedge e_{\ell_{3} m_{4}}$ if $k_{3} \neq \ell_{3}$. The other case is similar.

Fact. The elements in (6) are in $\operatorname{Im}\left(d_{3}\right)$.

Proof. $d_{3}\left(e_{i_{1} j_{2}} \wedge e_{j_{2} k_{3}} \wedge e_{k_{3} \ell_{4}}\right)=-e_{i_{1} k_{3}} \wedge e_{k_{3} \ell_{4}}+e_{i_{1} j_{2}} \wedge e_{j_{2} \ell_{4}}$. Such elements generate all elements as in (6).

Conversely, we must check $\operatorname{Im}\left(d_{3}\right) \subset \mathfrak{b}$. By straightforward verifications:

- $d_{3}\left(\mathfrak{u}_{14} \wedge \mathfrak{u} \wedge \mathfrak{u}\right) \subset \mathfrak{u}_{14} \wedge \mathfrak{u}$.

- $d_{3}\left(\mathfrak{u}_{13} \wedge \mathfrak{u}_{23} \wedge \mathfrak{u}_{24}\right)=0$

- $d_{3}\left(\mathfrak{u}_{12} \wedge \mathfrak{u}_{13} \wedge \mathfrak{u}_{24}\right), d_{3}\left(\mathfrak{u}_{13} \wedge \mathfrak{u}_{24} \wedge \mathfrak{u}_{34}\right), d_{3}\left(\mathfrak{u}_{12} \wedge \mathfrak{u}_{13} \wedge \mathfrak{u}_{34}\right), d_{3}\left(\mathfrak{u}_{12} \wedge \mathfrak{u}_{24} \wedge \mathfrak{u}_{34}\right)$ are all contained in $\mathfrak{u}_{14} \wedge \mathfrak{u}$.

- $d_{3}\left(\mathfrak{u}_{12} \wedge \mathfrak{u}_{13} \wedge \mathfrak{u}_{23}\right) \subset \mathfrak{u}_{13} \wedge \mathfrak{u}_{13}$, and similarly $d_{3}\left(\mathfrak{u}_{23} \wedge \mathfrak{u}_{24} \wedge \mathfrak{u}_{34}\right) \subset \mathfrak{u}_{24} \wedge \mathfrak{u}_{24}$.

- $d_{3}\left(\mathfrak{u}_{12} \wedge \mathfrak{u}_{23} \wedge \mathfrak{u}_{24}\right)$ and similarly $d_{3}\left(\mathfrak{u}_{13} \wedge \mathfrak{u}_{23} \wedge \mathfrak{u}_{34}\right)$ are contained in $\mathfrak{u}_{14} \wedge$ $\mathfrak{u}_{23}+\mathfrak{u}_{13} \wedge \mathfrak{u}_{24}$.

- The only remaining case is that of $\mathfrak{u}_{12} \wedge \mathfrak{u}_{23} \wedge \mathfrak{u}_{34}: d_{3}\left(e_{i_{1} j_{2}} \wedge e_{j_{2}^{\prime} k_{3}} \wedge e_{k_{3}^{\prime} \ell_{4}}\right)=$ $\delta_{k_{3} k_{3}^{\prime}} e_{i_{1} j_{2}} \wedge e_{j_{2}^{\prime} \ell_{4}}-\delta_{j_{2} j_{2}^{\prime}} e_{i_{1} k_{3}} \wedge e_{k_{3}^{\prime} \ell_{4}}$, which lies in (4) or in (6).

Finally $\operatorname{Im}\left(d_{3}\right)=\mathfrak{b}$.

It follows from Lemma 3.3 that $\operatorname{Ker}\left(d_{2}\right)=\mathfrak{h} \oplus \mathfrak{b}$. Since $\mathfrak{b}=\operatorname{Im}\left(d_{3}\right)$, this is a $D$-submodule. Let us check that $\mathfrak{h}$ is also a $D$-submodule; the computation will be used in the sequel.

The action of $S$ on $\mathfrak{u}$ by conjugation is given by

$$
\left(\begin{array}{cccc}
1 & 0 & 0 & 0 \\
0 & A & 0 & 0 \\
0 & 0 & B & 0 \\
0 & 0 & 0 & 1
\end{array}\right) \cdot\left(\begin{array}{cccc}
0 & X_{12} & X_{13} & X_{14} \\
0 & 0 & X_{23} & X_{24} \\
0 & 0 & 0 & X_{34} \\
0 & 0 & 0 & 0
\end{array}\right)=\left(\begin{array}{cccc}
0 & X_{12} A^{-1} & X_{13} B^{-1} & X_{14} \\
0 & 0 & A X_{23} B^{-1} & A X_{24} \\
0 & 0 & 0 & B X_{34} \\
0 & 0 & 0 & 0
\end{array}\right) .
$$

We must look at the action of $D$ on the elements in (1), (3), and (5). We fix $(A, B) \in D \subset S \simeq \mathrm{SL}_{n_{2}} \times \mathrm{SL}_{n_{3}}$, and we write $A=\sum_{j_{2}} a_{j_{2}} e_{j_{2} j_{2}}$ and $B=$ $\sum_{k_{3}} b_{k_{3}} e_{k_{3} k_{3}}$.

- (1):

$$
(A, B) \cdot e_{i_{1} j_{2}} \wedge e_{k_{1} \ell_{2}}=e_{i_{1} j_{2}} A^{-1} \wedge e_{k_{1} \ell_{2}} A^{-1}=a_{j_{2}}^{-1} a_{\ell_{2}}^{-1} e_{i_{1} j_{2}} \wedge e_{k_{1} \ell_{2}} .
$$

The action on the other elements in (1) has a similar form.

- (3) $\left(j_{2} \neq k_{2}\right)$ :

$$
(A, B) \cdot e_{i_{1} j_{2}} \wedge e_{k_{2} \ell_{3}}=e_{i_{1} j_{2}} A^{-1} \wedge A e_{k_{2} \ell_{4}} B^{-1}=a_{j_{2}}^{-1} a_{k_{2}} b_{\ell_{3}}^{-1} e_{i_{1} j_{2}} \wedge e_{k_{2} \ell_{3}} .
$$

The action on the other elements in (3) has a similar form.

- (5) $\left(\sum_{j_{2}} \alpha_{j_{2}}=0\right)$ :

$$
\begin{aligned}
(A, B) \cdot \sum_{j_{2}} \alpha_{j_{2}}\left(e_{i_{1} j_{2}} \wedge e_{j_{2} k_{3}}\right)= & \sum_{j_{2}} \alpha_{j_{2}}\left(e_{i_{1} j_{2}} A^{-1} \wedge A e_{j_{2} k_{3}} B^{-1}\right) \\
=\sum_{j_{2}} \alpha_{j_{2}} a_{j_{2}}^{-1}\left(e_{i_{1} j_{2}} \wedge a_{j_{2}} b_{k_{3}}^{-1} e_{j_{2} k_{3}}\right) & =b_{k_{3}}^{-1}\left(\sum_{j_{2}} \alpha_{j_{2}}\left(e_{i_{1} j_{2}} \wedge e_{j_{2} k_{3}}\right)\right) .
\end{aligned}
$$

The other case in (5) has a similar form. 
Lemma 3.6. 0 is not a weight for the action of $D$ on $H_{2}(\mathfrak{u})$.

Proof. As described in the proof of Lemma 3.2, we think of weights as elements of $M / P$. Hence, we describe weights as elements of $M=\mathbf{Z}^{n_{2}+n_{3}}$ rather than $M / P$, and must check that no weight lies in $P$.

(1) In (3.1), the weight is $-u_{j_{2}}-u_{\ell_{2}}$, hence does not belong to $P$ since $n_{2} \geq 3$. The other verifications are similar.

(3) In (3.2), the weight is $-u_{j_{2}}+u_{k_{2}}-v_{\ell_{3}}$, hence does not belong to $P$. The other verification for (3) is similar.

(5) In (3.3), the weight is $-v_{k_{3}}$, hence does dot belong to $P$. The other verification is similar.

Finally, Lemmas 3.2 and 3.6 imply that the conditions of Theorem 3.1 are satisfied, so that $\Gamma$ is finitely presented.

\section{ACKNOWLEDGMENTS}

I thank Herbert Abels, Yann Ollivier, and Frédéric Paulin for useful discussions, and Laurent Bartholdi and the referee for valuable comments and corrections.

\section{REFERENCES}

[A1] H. AbELS. An example of a finitely presentable solvable group. In: "Homological group theory," Proceedings Symposium, Durham, 1977, London Math. Soc. Lecture Note Ser. 36 (1979), 105-211. MR0564423 (82b:20047)

[A2] H. Abels. Finite presentability of S-arithmetic groups. Compact presentability of solvable groups. Lecture Notes in Math. 1261, Springer, 1987. MR0903449 (89b:22017)

[Be] H. BeHR. Chevalley groups of rank 2 over $\mathbf{F}_{q}(t)$ are not finitely presentable. In: "Homological group theory," Proceedings Symposium, Durham, 1977, London Math. Soc. Lecture Note Ser. 36 (1979), 213-224. MR0564424 (81e:20052)

[BHC] A. Borel, Harish-Chandra. Arithmetic subgroups of algebraic groups. Ann. of Math. $\mathbf{7 5}$ (1962), 485-535. MR0147566 (26:5081)

[BS] I. BelegradeK, A. SZCZEPAŃSKI. Endomorphisms of relatively hyperbolic groups. Preprint 2005, arXiv math.GR/0501321.

[Ha] P. HALl. The Frattini subgroups of finitely generated groups. Proc. London Math. Soc. (3) 11 (1961), 327-352. MR.0124406 (23:A1718)

[HV] P. DE LA HARPe, Alain VALETTE. La propriété (T) de Kazhdan pour les groupes localement compacts, Astérisque 175, SMF, 1989. MR1023471 (90m:22001)

[Kn] M. KNESER. Erzeugende und Relationen verallgemeinerter Einheitengruppen. J. Reine Angew. Math. 214/215 (1964), 345-349. MR0161863 (28:5067)

[Ma] G. Margulis. Discrete Subgroups of Semisimple Lie Groups. Springer, 1991. MR.1090825 (92h:22021)

[OW] Y. Ollivier, D. Wise. Kazhdan groups with infinite outer automorphism group. Preprint 2005, arXiv math.GR/0409203; to appear in Trans. Amer. Math. Soc.

[Pa] F. PAulin. Outer automorphisms of hyperbolic groups and small actions on $\mathbf{R}$-trees. In "Arboreal Group Theory" (MSRI, Berkeley, September, 1988) R.C. Alperin ed., M.S.R.I., Pub. 19, Springer-Verlag, 1991. MR.1105339 (92g:57003)

[RS] U. Rehrmann, C. SoulÉ. Finitely presented groups of matrices. In "Algebraic K-theory" Proceedings Conference, Evanston, 1976. Lecture Notes in Math. 551: 164-169, Springer, 1976. MR0486175 (58:5955)

[Sh] Y. Shalom. Bounded generation and Kazhdan's property (T). Publ. Math. Inst. Hautes Études Sci. 90 (1999), 145-168. MR.1813225 (2001m:22030)

[Wa] S. P. WANG. On the Mautner phenomenon and groups with property (T). Amer. J. Math. 104(6) (1982), 1191-1210. MR0681733 (84g:22033) 
[Wo] Report of the workshop Geometrization of Kazhdan's Property (T) (organizers: B. Bekka, P. de la Harpe, A. Valette; 2001). Unpublished; currently available at http://www.mfo. $\mathrm{de} /$ cgi-bin/tagungsdb?type=21\&tnr=0128a.

[Zu] A. ZuK. La propriété (T) de Kazhdan pour les groupes agissant sur les polyèdres. C. R. Math. Acad. Sci. Paris 323, Série I (1996), 453-458. MR.1408975 (97i:22001)

Institut de Géométrie, Algìbre et Topologie (IGAT), École Polytechnique Fédérale de Lausanne (EPFL), CH-1015 Lausanne, Switzerland

E-mail address: decornul@clipper.ens.fr 